\title{
PSO-based Secondary Frequency Control and Active Power Sharing
}

\author{
Mahmuda Begum, Li Li and Jianguo Zhu \\ Faculty of Engineering and Information Technology, University of Technology Sydney, Australia \\ Email: Mahmuda.Begum@ student.uts.edu.au
}

\begin{abstract}
A novel secondary control for restoring the system frequency in a distributed manner with incremental cost based droop-controlled microgrids (MGs) is presented in this paper. The proposed control ensures the frequency restoration and accurately share the active power in an optimal way simultaneously. Stability analysis with state space modelling is completed with the proposed distributed secondary frequency controller (DSFC). The DSFC parameters are designed with particle swarm optimization (PSO) technique where the selection of DSFC parameters are considered as an optimisation problem for enhancing overall stability of the system. A test MG model in the MATLAB based simcsape platform is taken for modelling and simulation. The outcomes from the simulation confirms that the proposed DSFC can simultaneously restore the frequencies and optimal active power sharing while the optimal DSFC parameters reduce the overshoot and maintain the better stability for the system.
\end{abstract}

Index Terms - Distributed Control, State Space Model, Consensus Control, Particle Swarm Optimisation.

\section{INTRODUCTION}

The main idea for designing a Microgrid (MG) is rapidly transforming from centralized to distributed ones where the generation and loads are structured as a whole part of multiple distributed MGs, or only distributed quantities in the future. Recent advancement in the MG control enhances the potential of MGs to be used in a greater level. The control system of autonomous MG is one of the crucial empowering technologies, which need to be designed accurately. Researchers are much more concerned in this area over the last few years. Hierarchical control structure for islanded MG systems has been recommended for the operational functionality of standard MG systems [1]. This hierarchical control consists of three level control, explicitly, primary, secondary and tertiary control level.

Primary control has the fastest response where droop control method is widely accepted as a practical solution for the primary control since it removes the communication requirement and considered as decentralized control. However, several limitations like frequency and voltage deviations (both steady-state and load-dependent), inaccurate reactive power sharing using droop control introduce the level of control hierarchy named secondary control. In traditional power system, centralised secondary controller is considered for the frequency and voltage restoration to their reference values. Control structures in distributed manner have been introduced to reduce the shortcomings of traditional integrated/central control systems. Secondary control structures in a distributed way have been initiated for the control of MGs mainly to reduce the system's cost and complexity while contribute more in dynamic performance of the system. Typically distributed control methods are based on multi-agent system or consensus protocols [2, 3]. Distributed control based on consensus algorithm exhibits a good performance utilising a sparse communication system.

However, dynamic behavior of MG with DSC is responsible for introducing the unwanted characteristics related to the system damping, response time, and stability region. Transfer function [4] based approach, state-space modeling and smallsignal analysis with initial condition and estimating the eigenvalues in time domain, and the impedance-based method in frequency domain [5] are the most common techniques for the stability analysis in detail. The main advantages of impedance analysis and the eigenvalue analysis methods are reducing the computation time while controller dynamics and the grid impedance are taken into account. The latter two methods are therefore more appropriate for the stability analysis with power electronics interfaced power systems. In this paper, detail stability analysis with state-space modelling based on the eigenvalue-analysis approach is chosen to see the effect of proposed Distributed Secondary Frequency Control (DSFC) on the overall system stability.

Furthermore, another key concern need to be further researched which is based on cost minimisation. The MG control system should be designed to get the economic benefit and stability of the MG while regulating the active power balance. Most of the prior works only considered the case that power outputs(both active and reactive) from inverters can proportionally share the total load in accordance with their power ratings [6]. An optimal amount of output power, combination of all generators is a suitable solution for lowering the operational cost and at the same time retaining system generation demand steadiness constraints. There are some active power control systems for cost minimisation have been proposed in last few decades. In [7], using equal increment cost theory based droop control approach is suggested to reduce the overall generation budget. However, the optimal control parameters are not taken into account. The work in [8] offered an active power sharing control arrangement both in distributed and optimal way based on equal incremental cost condition where they utilise the distributed approach for minimising the total generation costs of the system while the he detail stability analysis is not taken into consideration. The key impacts of this paper are:

i. Restoring the system frequency at the nominal value $\omega_{\text {ref }}$ within finite time.

ii. Minimising the operation cost by applying the optimal active power sharing using the equal cost increment values of 
Distributed Generation (DG) units, i.e., $\eta_{1}\left(P_{1}\right)=\eta_{2}\left(P_{2}\right)=$ $\cdots=\eta_{N}\left(P_{N}\right)$ where $\eta_{i}\left(P_{i}\right)$ denotes the cost incremental value function of $i^{\text {th }}$ DG unit.

iii. The stability analysis represents the effect of DSFC parameters on system stability.

iv. Utilising optimisation technique Particle Swarm Optimisation (PSO), the proposed DSFC shows better convergence and stability performance through the optimal control parameters.

The paper is organised as follows: In Section II, the design of proposed DSFC is represented. In Section III, the state space model of a test MG considering DSFC is explained. Modeling of the network and load is also given in this section. In Section IV, the stability analysis considering state space model with the help of eigenvalue analysis is shown. Section V describes the optimisation technique with PSO and lastly, the conclusion is presented in Section VI.

\section{II.DISTRIBUTED SECONDARY CONTROL}

This section familiarises the conception of distributed control algorithm in the controller design for MG based on the proposed secondary frequency restoration scheme. The secondary control selects the accurate primary control reference for frequency synchronisation. In relation to the distributed control theory, the secondary control objectives are proposed and then a DSFC law is designed for the MG to restore the deviated frequencies to the reference values. In the distributed control environment [2], a consensus problem is considered as one of the most vital and difficult problems. From a general point of view, the MG acts as a multi-agent system where every DG works as an agent in the consensus control. Agents are able to exchange the required data/information with their neighbours by simple and less expensive communication network to achieve the consensus. In this section, some basics of graph theory and consensus based recommended DSFC are discussed.

\section{A. Preliminaries}

\section{1) Graph Theory}

A directed graph (digraph) $G=\left(N_{G}, E_{G}\right)$ with a set of $N$ nodes, $N_{G}=\{1,2,3,4 \ldots \ldots . N\}$, a set of edges $E_{G} \subset N_{G} \times N_{G}$ and an adjacency matrix $A_{G}=\left(a_{i j} \geq 0\right) \in R^{N \times N}$ (where $a_{i j}=$ 1 if there is a path from the $i^{\text {th }}$ node to the $j^{\text {th }}$ node and otherwise $a_{i j}=0$ ) is considered here. An agent is represented by each node, and each edge $(i, j)$ (pointing from $j$ to $i$ ) indicates that the information flows from $j$ to $i$ related with $a_{i j}$. The neighbors of node $i$ is represented as $N_{i}=\{j \in$ $\left.N_{G}:(i, j) \in E_{G}\right\}$. According to this, an agent/node $i$ only has access to the data from its neighbors in $N_{i}$. If every agent (node) can be represented as a single-state system defined by $\dot{x}_{l}=u_{i}$ where $u_{i}$ is the input as a function of the $i^{\text {th }}$ agent's neighboring state $x_{j}, j \in N_{i}$, the common form of consensus protocol is as below:

$$
\dot{x_{\imath}}=u_{i}=-\sum_{j \in N_{i}} a_{i j}\left(x_{i}-x_{j}\right)
$$

\section{B. Droop Based DG unit}

Generally in an MG system, every inverter interfaced DG comprises a primary constant dc power source, a voltage source inverter (VSI), an LCL filter (combining the LC filter with coupling inductor) and output connector as shown in Fig. 1. A test MG model of three DG units is considered here which are connected in parallel. Every DG is associated with the load and linked with neighboring DG through feeder lines. The power controller (Fig. 1) permits DG units to share the active and reactive power requirement according to their maximum ratings based on the droop gain, i.e.,

$$
\begin{aligned}
\omega_{i} & =\omega_{\text {ref }}-m_{p i} P_{i} \\
v_{i} & =v_{\text {ref }}-n_{q i} Q_{i} \ldots
\end{aligned}
$$

where, $P_{i}$ and $Q_{i}$ are the measured active and reactive power at the output of $i^{t h}$ DG units, respectively; $m_{p i}$ and $n_{q i}$ are the droop coefficient for frequency and voltage control of $i^{\text {th }}$ DG units, respectively; $\omega_{\text {ref }}$ and $v_{\text {ref }}$ are the reference angular frequency and reference voltage, respectively.

\section{Formulation of Control Algorithm in a Distributed} and Optimal way

Distributed optimal control algorithm is untaken in this section as a resolution of economic dispatch (ED) problem which suggests a technique to minimise operation costs of the system. In general, if there are $n$ DGs, an ED problem is welldefined as follows:

$$
\begin{aligned}
\text { Min } & F=\sum_{i=1}^{n} F_{C i}\left(P_{i}\right) \\
\text { s.t. } & \sum_{i=1}^{n} P_{i}=P_{\text {load }},
\end{aligned}
$$

where $P_{i}^{\min } \leq P_{i} \leq P_{i}^{\max } ; P_{\text {load }}$ is the entire load demand satisfying $\sum_{i=1}^{n} P_{i}^{\text {min }}<P_{\text {load }}<\sum_{i=1}^{n} P_{i}^{\text {max }} ; P_{i}^{\text {min }}$ and $P_{i}^{\text {max }}$ are the minimum and maximum output of $i^{\text {th }} \mathrm{DG}$, respectively.

For traditional diesel generators, the cost function $F_{C i}\left(P_{i}\right)$ is expressed as a convex quadratic function of the outputs of active power as,

$$
F_{C i}\left(P_{i}\right)=q_{i} P_{i}^{2}+r_{i} P_{i}+s_{i}
$$

where $q_{i} \geq 0, r_{i}>0$ and $s_{i}>0$, and they are all the cost parameters of $D G_{i}$.

The cost parameters used in the generation cost function $F_{C i}\left(P_{i}\right)(\$ / \mathrm{kW})$ of each DG unit are given in [9]. References [79] suggest a droop-based control to offer the optimal active power sharing for DG units which is based on the equal increment cost principle (ECP) to minimise the total generation costs $\left(F_{C i}\left(P_{i}\right)\right)$ of the MG, as specified by

$$
\begin{aligned}
& \omega_{i}=\omega_{\text {ref }}-e \eta_{i}\left(P_{i}\right) \ldots \ldots \ldots \ldots \ldots \\
& \eta_{i}\left(P_{i}\right)=\frac{d\left(G C_{i}\left(P_{i}\right)\right)}{d P_{i}}=2 q_{i} P_{i}+r_{i}
\end{aligned}
$$

In (5), $e$ is a scalar positive quantity. The frequency $\omega_{i}$ of all DG units must be same at the steady-state condition. Therefore, $\eta_{i}\left(P_{i}\right)$ 's are also same for all the DG units, which fulfils the ECP of the ED. In this paper, to get the optimal active power, (5) instead of (1) for the active power sharing is used.

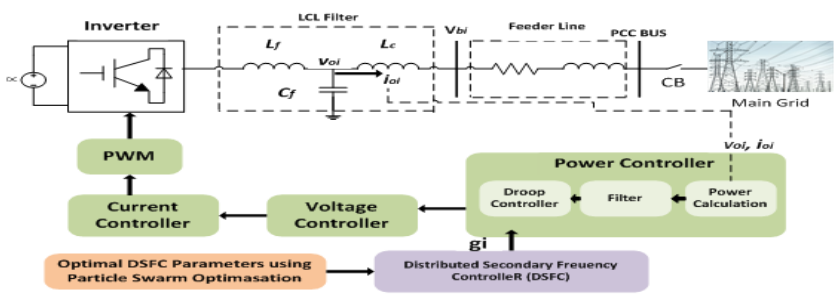

Fig. 1. Simplified structure of an inverter-based DG (primary and DSFC) 
The secondary control is accomplished by selecting the frequency magnitude $\omega_{i}$ to the reference $\omega_{\text {ref }}$ synchronously with all the agents performing as a group. Hence, the consensus based DSFC signal for the $i^{\text {th }}$ DG can be written as follows:

$$
\begin{gathered}
g_{i}=-C_{\omega} \int\left[\sum_{j \in N_{i}} a_{i j}\left(\omega_{i}-\omega_{j}\right)+b_{i}\left(\omega_{i}-\omega_{r e f}\right)\right]- \\
C_{p} \sum_{j \in N_{i}} a_{i j}\left(e \eta_{i}\left(P_{i}\right)-e \eta_{j}\left(P_{j}\right)\right) \ldots \ldots \ldots \ldots \ldots \ldots \ldots \ldots \ldots \ldots \ldots \ldots \ldots \ldots \ldots \ldots \ldots \ldots
\end{gathered}
$$

where $g_{i}$ is the secondary control signal both for frequency restoration and optimal active power sharing. It should be noted that, in (7), $b_{i}=1$ if the $i^{\text {th }} \mathrm{DG}$ is directly connected with the controller at the point of common coupling and otherwise $b_{i}=$ 0 . Adding the control signals in (5), we get the final inverter frequency shown below:

$$
\omega_{i}=\omega_{\text {ref }}-e \eta_{i}\left(P_{i}\right)+g_{i}
$$

\section{State Space Modelling OF Test Microgrid}

The entire test MG model is considered for state-space modelling where inverter, network and load are modelled as 3 main sub-groups as in [6].

\section{A. Modelling of Single Inverter}

Rotational frequency of an inverter is determined by its local controller and every inverter has its own reference frame. In this paper, dynamics are considered for modelling an inverter comprises the power controller, output filter (combining LC filter and coupling inductor) and voltage and current controller (Fig.1). Small-signal modeling of inverters connected in parallel is done while the network and load dynamics are in consideration as well. Here, state equations of the lines and loads are represented on the common reference frame. All the inverters, lines and loads are transformed to this common reference frame via the park transformation [6]. Here, the axis set $(D-Q)$ is the common reference frame rotating at a frequency $w_{\text {com }}$, whereas the axis $(d-q)_{i}$ is the reference frame of the $i^{\text {th }}$ inverter rotating at $\omega_{i}$.

$$
\text { Now, } \quad\left[f_{D Q i}\right]=\left[T_{i}\right]\left[f_{d q i}\right] \ldots \ldots(9),\left[T_{i}\right]=\left[\begin{array}{cc}
\cos \left(\delta_{i}\right) & -\sin \left(\delta_{i}\right) \\
\sin \left(\delta_{i}\right) & \cos \left(\delta_{i}\right)
\end{array}\right] \ldots
$$

where $\delta_{i}$ is the angle between the common reference frame and the reference frame of $i^{t h}$ inverter. The detailed equations of modelling of all these sub-groups can be found in [6] considering the primary droop control. In the following sections, only 3-phase voltages and currents are considered as vector quantity in $D-Q$ reference frame and other parameters are consider as scalar quantity.

\section{B. Modelling of Single Inverter considering DSFC}

According to [6], the resulting small-signal linearized statespace model of an individual inverter unit in the common reference frame considering the primary control (droop control) only can be written as below (the detail specifications can be found in [7]):

$$
\begin{gathered}
{\left[\Delta \dot{x}_{l}\right]=\left[A_{i}\right]\left[\Delta x_{i}\right]+\left[B_{i}\right]\left[\Delta v_{b D Q i}\right]+\left[C_{i}\right]\left[\Delta \omega_{c o m}\right]} \\
{\left[\begin{array}{c}
\Delta \omega_{i} \\
\Delta i_{0 D Q i}
\end{array}\right]=\left[\begin{array}{c}
D_{\omega i} \\
D_{c i}
\end{array}\right]\left[\Delta x_{i}\right]}
\end{gathered}
$$

here $\quad, \Delta x_{i}=\left[\Delta \delta_{i}, \Delta P_{i}, \Delta Q_{i}, \Delta \emptyset_{d q i}, \Delta \gamma_{d q i}, \Delta i_{l d q i}, \Delta v_{0 d q i}, \Delta i_{0 d q i}\right]^{T}$; $\emptyset_{d q i}$ and $\gamma_{d q i}$ are the state variables related to voltage and current controller respectively; $i_{l d q i}$ represents the state variables of filter current; $v_{0 d q i}$ and $i_{0 d q i}$ represent the state variables of inverter output voltage and current respectively.

In accordance with this, the proposed DSFC can be added with primary control and forms the following small signal linearised state-space equation for an inverter considering control (from (1)-(8)) in the common reference frame:

$$
\begin{aligned}
& {\left[\Delta \dot{x}_{i}\right]=\left[A_{i}\right]\left[\Delta x_{i}\right]+\left[B_{i}\right]\left[\Delta v_{b D Q i}\right]+\left[C_{i w}\right]\left[\Delta w_{c o m}\right]+\sum_{j \in N_{i}}\left[F_{i j}\right]\left[\Delta x_{j}\right] \ldots \text { (11) }} \\
& {\left[\begin{array}{c}
\Delta \omega_{i} \\
\Delta i_{0 D Q i}
\end{array}\right]=\left[\begin{array}{c}
D_{\omega i} \\
D_{c i}
\end{array}\right]\left[\Delta x_{i}\right]}
\end{aligned}
$$

In (11), the state variables of each $D G$ unit are considered as $\Delta x_{i}=\left[\Delta \delta_{i}, \Delta P_{i}, \Delta Q_{i}, \Delta g_{i}, \Delta \emptyset_{d q i}, \Delta \gamma_{d q i}, \Delta i_{l d q i}, \Delta v_{0 d q i}, \Delta i_{0 d q i}\right]^{T}$.

In (11) \& (12), $\left[A_{i}\right],\left[B_{i}\right],\left[C_{i w}\right],\left[F_{i j}\right],\left[D_{\omega i}\right]$ and $\left[D_{c i}\right]$ are the parameter matrices. $\left[F_{i j}\right]$ indicates the correlation between $D G_{i}$ and $D G_{j}$. [ $\left.\Delta v_{b D Q i}\right]$ denotes the deviation of bus voltages. [ $\left.\Delta i_{0 D Q i}\right]$ shows the deviations of inverter output currents. The equation for new states of secondary controller can be written as

$\Delta g_{i}=-C_{w} \int\left[\sum_{j \in N_{i}} a_{i j}\left(\Delta \omega_{i}-\Delta \omega_{j}\right)+b_{i} \Delta \omega_{i}\right]-C_{p}\left[\sum_{j \in N_{i}} a_{i j} e\left(2 q_{i} \Delta P_{i}-\right.\right.$ $\left.\left.2 q_{j} \Delta P_{j}\right)\right]$ ).

\section{Combined State-Space Model of all the Inverters}

Three DGs connected in parallel are considered in the proposed model. Now, by combining (9)-(13) for 3 inverters, the resulting small-signal model of all the inverter units is obtained, as shown below:

$$
\begin{aligned}
& {[\dot{\Delta x}]=[A][\Delta x]+[B]\left[\Delta v_{b D Q}\right]} \\
& {\left[\begin{array}{c}
\Delta \omega \\
\Delta i_{0 D Q}
\end{array}\right]=\left[\begin{array}{c}
D_{\omega} \\
D_{c}
\end{array}\right][\Delta x] \ldots \ldots \ldots \ldots . .}
\end{aligned}
$$

To get the combined model for the system, the sub-model of all the individual DG inverters along with the network and individual load models need to be combined in the common reference frame.

\section{Network Modelling}

Small-signal linearized state space model of the network sub-module including two RL type lines can be given in the common reference frame [6] as shown below:

$$
\left[\Delta \dot{l}_{l c D Q}\right]=\left[A_{n c}\right]\left[\Delta i_{l c D Q}\right]+\left[B_{n c}\right]\left[\Delta v_{b D Q}\right]+\left[C_{n c}\right]\left[\Delta \omega_{c o m}\right] .
$$

$\left[\Delta i_{l c D Q}\right]$ denotes the deviations of line current in the network.

$$
\begin{aligned}
& \quad A_{n c}=\operatorname{diag}\left(A_{n c 1}, A_{n c 2}\right) ; B_{n c}=\left[\begin{array}{ccc}
B_{n c 1} & -B_{n c 1} & 0 \\
0 & B_{n c 2} & -B_{n c 2}
\end{array}\right], \\
& B_{n c i}=\operatorname{diag}\left(L_{l c i}{ }^{-1}, L_{l c i}{ }^{-1}\right) ; C_{n c=}\left[C_{n c 1}, C_{n c 2}\right]^{T} ; C_{n c i}=\left[I_{l c Q i},-I_{l c D i}\right]^{T} ; \\
& \text { E. Load Modelling }
\end{aligned}
$$

Small-signal linearized state-space model of the RL type load sub-module can be written [6] as in (17). $\Delta i_{\text {loadcDQ }}$ denotes the deviations of load current.

$$
\begin{gathered}
{\left[\Delta u_{\text {loadcDQ }}\right]=\left[A_{\text {lo }}\right]\left[\Delta i_{\text {loadcDQ }}\right]+\left[B_{\text {loadc }}\right]\left[\Delta v_{\text {bDQ }}\right]+\left[C_{\text {loadc }}\right]\left[\Delta \omega_{\text {com }}\right] . .} \\
A_{\text {loadc }}=\operatorname{diag}\left(A_{\text {loadc1 }}, A_{\text {loadc } 2}\right) ; \\
B_{\text {loadc }}=\operatorname{diag}\left(B_{\text {loadc } 1}, B_{\text {loadc } 2}\right) ; \\
C_{\text {loadc }}=\left[C_{\text {loadc1 } 1}, C_{\text {loadc } 2}\right]^{T}, B_{\text {loadci }}=\operatorname{diag}\left(L_{\text {loadci }}{ }^{-1}, L_{\text {loadci }}{ }^{-1}\right) \\
C_{\text {loadci }}=\left[I_{\text {loadcQi }},-I_{\text {loadcDi }}\right]^{T}
\end{gathered}
$$

Denote the deviation of $i_{o i}$, of all the DG units as $\left[\Delta i_{o D Q}\right]$. Then the bus voltage, $\Delta v_{b D Q}$ is represented by

$$
\left[\Delta v_{b D Q}\right]=R_{N}\left(M_{I}\left[\Delta i_{o D Q}\right]+M_{N}\left[\Delta i_{l c D Q}\right]+M_{L}\left[\Delta i_{\text {loadcDQ }}\right]\right) \text {. }
$$


TABLE I. INVERTERS PARAMETERS USED IN THE TEST MG

\begin{tabular}{|c|c|c|c|}
\hline Description & Parameter & Value & Unit \\
\hline \multicolumn{4}{|c|}{ Parameters for Microgrid Modelling } \\
\hline DC Bus Voltage & $V_{d c}$ & 700 & $\mathrm{~V}$ \\
\hline Nominal Voltage & $v_{\text {ref }}$ & 311 & V \\
\hline Nominal Frequency & $f_{\text {ref }}$ & 50 & $\mathrm{~Hz}$ \\
\hline Filter Resistance & $R_{f}$ & 0.1 & $\Omega$ \\
\hline Filter Inductance & $L_{f}$ & 1.35 & $\mathrm{mH}$ \\
\hline Filter Capacitance & $C_{f}$ & 50 & $\mu \mathrm{F}$ \\
\hline Coupling Inductor & $R_{c}$ & 0.03 & $\Omega$ \\
\hline Resistance & & & \\
\hline Coupling Inductor & $L_{C}$ & 0.1 & $\mathrm{mH}$ \\
\hline Inductance & & & \\
\hline \multicolumn{4}{|c|}{ Parameters for Voltage Control } \\
\hline Proportional Voltage Gain & $K_{P v}$ & 0.05 & \\
\hline Integral Voltage Gain & $K_{I v}$ & 390 & \\
\hline Feed Forward Gain & $\mathrm{F}$ & 0.75 & \\
\hline \multicolumn{4}{|c|}{ Parameters for Current Control } \\
\hline Proportional Current Gain & $K_{P c}$ & 10.2 & \\
\hline Integral Current Gain & $K_{I c}$ & $16 \mathrm{e} 3$ & \\
\hline
\end{tabular}

The detailed specifications of (18) can be found in [7]. The complete MG state-space model and the resulting system matrix is achieved by utilising the individual sub-group models given by (14)-(18) as in (19). The assessment of complete system dynamics and stability performance considering the eigen-analysis of system matrix is given in the following sections.

$$
\left[\begin{array}{c}
\Delta x \\
\Delta l_{\text {lcl }} \\
\Delta l_{\text {loadc }}
\end{array}\right]=A_{M G}\left[\begin{array}{c}
\Delta x \\
\Delta i_{\text {lci }} \\
\Delta i_{\text {loadc }}
\end{array}\right]
$$

\section{STABILITY ANALYSIS}

\section{A. Test Microgrid Model}

A test MG of three inverter-based DG units including the local loads as in Fig. 2, is considered here for analysis through several case studies. The microgrid presented here is a $311 \mathrm{~V}$

TABLE II SPECIFICATIONS OF FEEDER LINE AND LOAD

\begin{tabular}{ccc|ccc}
\hline \hline \multicolumn{3}{c|}{ Line Data } & \multicolumn{3}{c}{ Load Data } \\
\hline \hline No. & $\mathrm{R}(\Omega)$ & $\mathrm{L}(\mu \mathrm{H})$ & No. & $\mathrm{R}(\Omega)$ & $\mathrm{L}(\mathrm{mH})$ \\
Line1 & 0.23 & 318 & Load1 & 80 & 55 \\
Line2 & 0.30 & 312 & Load2 & 70 & 22
\end{tabular}

and $50 \mathrm{~Hz}$ system, operated in islanded mode and is simulated in MATLAB. The initial conditions around an operating point are found from the time-domain simulation of test MG model. DG1 and DG3 are connected to Load1 and Load 2 respectively through the coupling inductance $L_{c}(0.1 \mathrm{mh})$ as in Fig. 2 . The load is chosen as a 3-phase series RL load and each feeder is designed as a series RL branch in every phase. Tables I, II and IV provide the specifications of the system, DG primary and secondary controller, respectively.

A directed graph as in Fig. 2 (blue dashed line) is considered for the communication among DG units. For the frequency restoration problem, DG2 output is taken as the reference one. Fig. 2 also indicates that (i) all the DGs like can be considered as root nodes, and (ii) DG2 is the leader node which provides the reference value and the pinning gain $b_{2}=1$. The results can be divided into two parts. One is eigenvalue analysis, and another is time domain simulation; the results from both are analysed with and without optimisation implementation.

\section{B. Eigenvalue Analysis}

This subsection presents the system dynamic behaviour and small-signal stability analysis along with the proposed DSFC. The eigenvalues of the system matrix $A_{M G}$ can be used to assess the system stability around the equilibrium. According to the established small-signal model in the previous section, the resulting eigenvalues considering DSFC are shown in Fig. 3 for stable operation. The main emphasis for stability analysis is on the low-frequency modes as the eigenvalues of high and

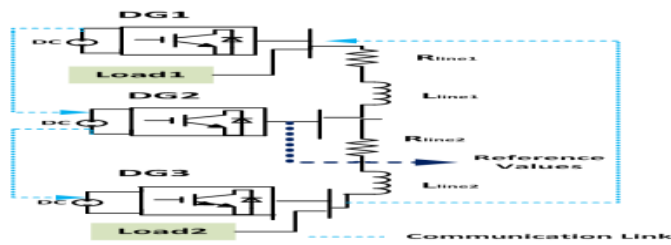

Fig. 2. MG test model for simulation and the communication diagraph

intermediate frequency modes have weak influence on the system stability [6]. The leading less damped modes are shown in Fig. 6(a) which indicates that the DSFC significantly participate for creating the shape of the eigenvalues on the complex plane which introduces a new pair of oscillatory modes making less damping responses as compared with the primary responses [10]. Therefore, the secondary control needs to be designed accurately for getting the stable system operation.

1) Participation Factors: $\Delta \delta_{i}$ and $\Delta P_{i}$ are major participants states when considering only primary droop control for the lowfrequency modes according to the participation factor analysis [6]. The details of participation factor analysis can be found in [7]. From Fig. 6(a), it is clear that modes 1-6 are affected by the proposed DSFC state $\Delta g_{i}$ along with $\Delta \delta_{i}$ and $\Delta P_{i}$.

2) Eigenvalue Sensitivity to Secondary Control Parameters on System Stability: In this paper, the sensitivity analysis is done considering the secondary control parameters $C_{\omega}$, and $C_{p}$. The effects of the secondary control parameters on the system stability are shown in Fig. 4. Figs. 4(a-b) show the traces of the system's low-frequency modes (modes 1-6) as functions of $C_{\omega}$ and $C_{p}$. Fig. 4(a) and Fig. 4(b) present variations in the damping

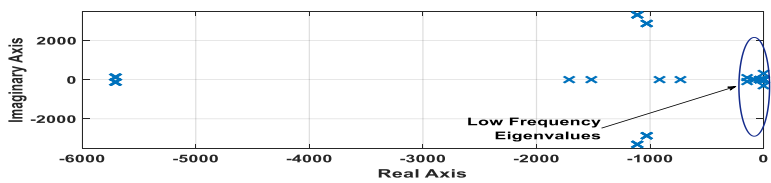

Fig. 3. Eigenvalues of system matrix considering DSFC for stable operation of the oscillatory modes when $C_{\omega}$ and $C_{p}$ both vary from 0 to 100 and 0 to 1000 respectively. These variations occurs in modes 1-6. Fig. 4(a) and Fig. 4(b) show that, (i) for a given value of $C_{p}$, the stability is increased when $C_{\omega}$ increases, and (ii) for a particular value of $C_{\omega}$, the oscillation increases when $C_{p}$ increases. DSFC parametrs can be chosen based on the above analysis which gives a general idea about designing DSFC parametrs as well. 


\section{DSFC OPTIMISATION USING PSO}

The stability analysis given in Section IV shows that DSFC parameters can greatly affect the system stability. Therefore, the optimal values of DSFC parameters are needed. The main goal of the optimisation problem chosen here is to enhance the system stability by choosing the accurate position of eigenvalues. Eigenvalues are on the left side of imaginary axis make the system stable and stability increases with the distance from imaginary axis increases. Denote the eigenvalues as, $\lambda_{i}=$ $R e_{i}+j I m g_{i}$, where $R e_{i}$ and $I m g_{i}$ are the real and imaginary part of the eigenvalue, respectively. So the objective function is Min $\mathrm{J}=$ maximum of $\left(R e_{i}\right)$ such that $C_{\omega}, C_{p}$ are within the limit (Table III). According to the general principle of PSO, each particle is a real valued vector with dimension equal to the number of parameters to be optimized. Each particle is one solution to the defined optimisation problem and the size of the population named as $\mathrm{P}$ particles will be there. Firstly a random solution is considered which is the position in the search space within the parameter limit. The position of each particle is updated based on its inertia, personal best and group best consistent with the equation stated below $V_{i}^{k+1}=w \cdot V_{i}^{k}+C_{1} \cdot r_{1}\left[X_{\text {pbest }}^{k}-X_{i}^{k}\right]+C_{2} \cdot r_{2}\left[X_{\text {gbest }}^{k}-X_{i}^{k}\right]$

$$
X_{i}^{k+1}=X_{i}^{k}+V_{i}^{k+1}
$$

where, $X_{i}^{k}$ and $V_{i}^{k}$ are the position and velocity of $i^{t h}$ particle in $k^{\text {th }}$ iteration, respectively; $w$ is the coefficient of inertia; $C_{1}$ and $C_{2}$ are the coefficients of acceleration; $X_{\text {pbest }}^{k}$ and $X_{g b e s t}^{k}$ are the local and global best position of $i^{t h}$ particle in $k^{t h}$ iteration.

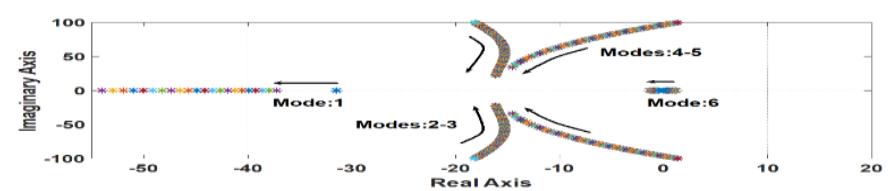

(a) $C_{\omega}$ increases from 0 to 100

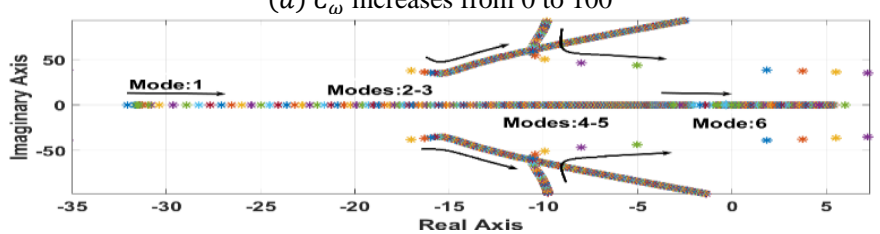

(b) $C_{p}$ increases from 0 to 1000

Fig. 4. Traces of low frequency modes (a) \& (b) Modes 1-6

The optimum value is obtained by performing this updating method for velocity and position at the end of a few iterations. The parameters chosen for the optimisation problem discussed earlier, are given in Table III.

TABLE III. PARAMETERS USED FOR PSO

\begin{tabular}{c|c}
\hline \hline Parameter & Values \\
\hline \hline Maximum Iteration & 100 \\
Swarm Size & 70 \\
Coefficient of Inertia & 1 \\
Damping Ratio of Inertia Coefficient & 0.99 \\
Coefficient of Local Acceleration & 2 \\
Coefficient of Global Acceleration & 2 \\
Unknown Variables & {$\left[C_{\omega}, C_{p}\right]$} \\
Lower Limit of Unknown Variables & {$[0,100]$} \\
Upper Limit Unknown Variables & {$[0,1000]$}
\end{tabular}

By utilising the optimisation discussed above for the DSFC parameters $\left[C_{\omega}, C_{p}\right]$, the optimal values are obtained as $[50.459$, 620.39] and the $\mathrm{J}=-30.06$. The convergence curve of objective function is shown in Fig. 5. The differences of dominant eigenvalues before and after optimisation are given in Fig. 6(a) and Fig. 6(b). In Fig. 6(a), the real part of extreme right eigenvalue is at -5.001 and it is moved to -30.06 after the optimization as in Fig. 6(b). Therefore, stability can be enhanced by selecting the optimal values of control parameters $C_{\omega}, C_{p}$ for the proposed DSFC. The optimal values for the DSFC are summarised in Table IV.

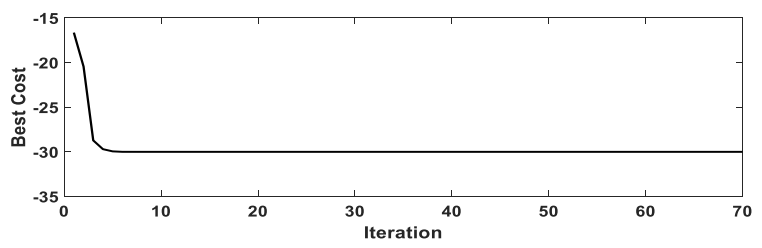

Fig. 5. Convergence curve of objective function

TABLE IV. SECONDARY CONTROLLER PARAMETERS

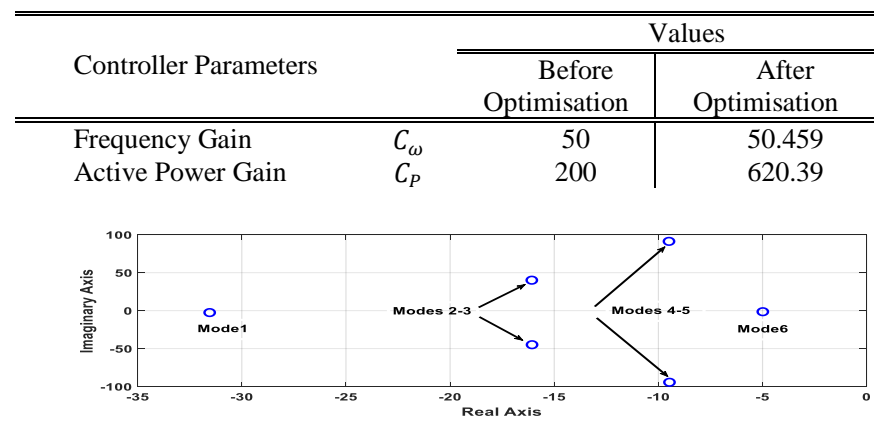

(a)

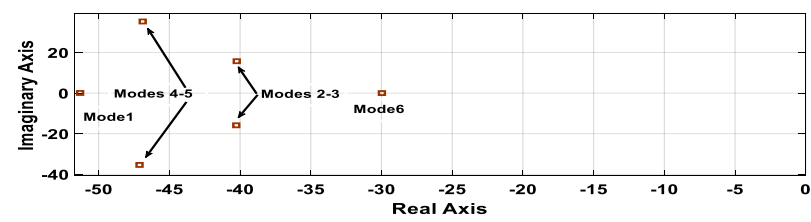

(b)

Fig. 6. Dominant eigenvalues (a) before and (b) after optimisation in S plane

\section{TIME-DOMAIN SIMULATION AND DISCUSSION}

The performance of the proposed DSFC are validated by the time-domain simulation with two case studies performed in the Matlab/Simpower system platform.

A. Case 1(DSFC without PSO optimisation): The performance of primary control (5) is shown in Figs. 7(a) and (b). Fig. 7(a) indicates due to the primary control, the frequencies of 3 DGs deviate to $49.88 \mathrm{~Hz}$. Thus, the primary control exhibits a clear deviation of frequencies from the nominal value, $50 \mathrm{~Hz}$, which must be restored to their rated values by applying the secondary control. Fig. 8(a) shows that the proposed DSFC can give the fast response for restoring the deviated frequencies to $50 \mathrm{~Hz}$. Moreover, Fig. 7(b) indicates that the active power sharing is based on their capacities with the ratio $P_{1}: P_{2}: P_{3}=2: \frac{3}{2}: 1$ for the primary control only. Fig. 8 (c) shows the incremental costs, $\eta_{i}\left(P_{i}\right)$, converge to the same value $\$ 4.56 / \mathrm{kW}$. That is, proposed DSFC imposes the reference optimal active power sharing according to the cost function to be $P_{1}=24 \mathrm{~kW}, P_{2}=29 \mathrm{~kW}$ and $P_{3}=21 \mathrm{~kW}$ as in Fig. $8(\mathrm{~b})$. 


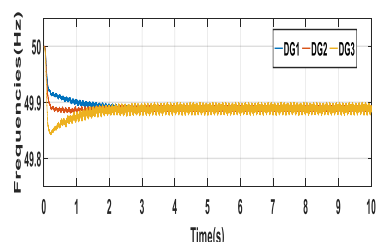

(a)

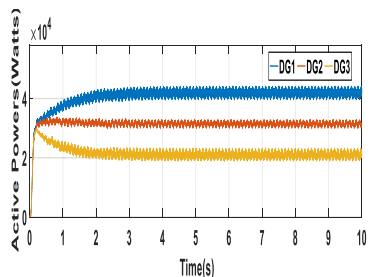

(b)
Fig. 7. Output of DGs under only primary control (Droop Control) (a) Frequencies (b) Active Powers

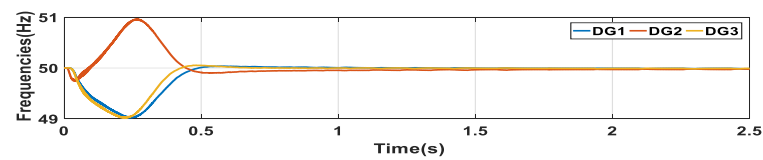

(a)

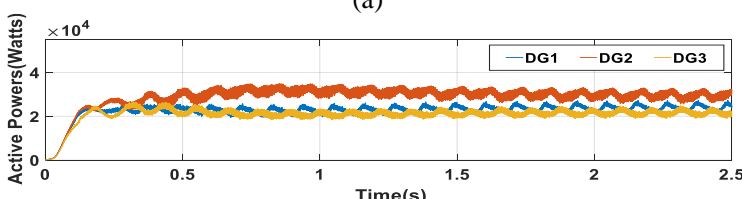

(b)

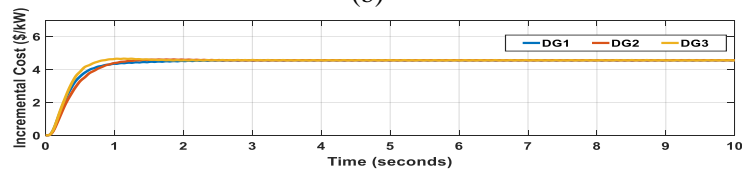

(c)

Fig. 8. Output of DGs with applying DSFC (a) Frequencies (b) Optimal values for active powers (c) Incremental cost values

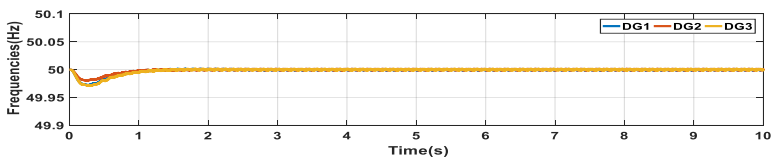

(a)

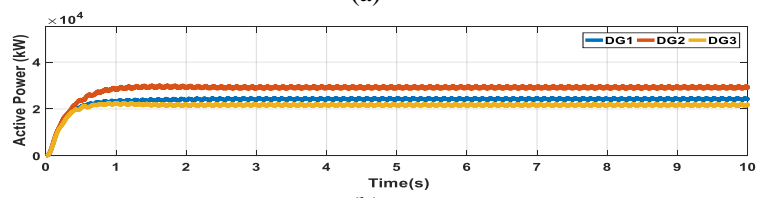

(b)

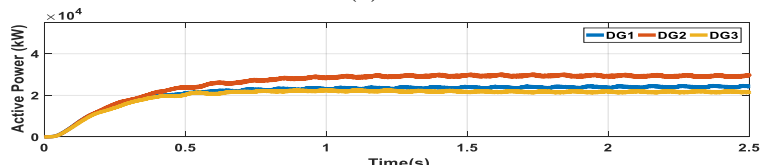

(c)

Fig. 9. Output of DGs with DSFC after PSO optimization (a) Frequencies (b) Optimal values for active powers for 10s (c) Optimal values for Active Powers for $2.5 \mathrm{~s}$

\section{B. Case 2 (DSFC with PSO optimal values after reaching} nominal values):

To show the effectiveness of DSFC after PSO optimisation, the outcomes from the simulation are given in Fig. 9. The results indicate that optimal control parameters can reduce the overshoot of frequencies and active power outputs compared with Fig. 8 while maintaining the same optimal active power sharing. Moreover, the corresponding convergence is increased (less than 1s) in Fig. 9(a) compared with Fig. 8(a) (approximately $1.5 \mathrm{~s}$ ), which indicates the corresponding settling time is decreased.

\section{CONCLUSION}

This paper presents a DSFC based on the cost function optimization along with the detail stability analysis. The stability analysis based on eigenvalue analysis reveals that the system stability may be reduced by the DSFC which adds oscillatory modes. An appropriate optimsation method is needed to get the system operate in a more stable condition. In this paper, the PSO algorithm is utilised for designing the optimal control parameters for DSFC. The proposed DSFC can restore the system frequencies to their nominal values. The resulting system can converge to the optimal reference values for active power sharing which are given by the cost function and also maintain that values in the steady state condition. The outcomes from the eigenvalue study and time-domain simulation ensure that the system stability of the test MG model is improved after applying the proposed optimal control parameter design method. As there are inadequate approaches for solving the problems of frequency recovery with optimal active power sharing in a distributed way, this research presents a DSFC with optimal active power sharing along with stability analysis and optimisation for DSFC parameters. These analysis can be used for designing the DSFC for frequency recovery and active power control in a distributed and optimal way.

\section{REFERENCES}

[10] M. Begum, L. Li, J. Zhu, and Z. Li, "State-Space Modeling and Stability Analysis for Microgrids with Distributed Secondary Control," in 2018 IEEE 27th International Symposium on Industrial Electronics (ISIE), 2018, pp. 1201-1206. 